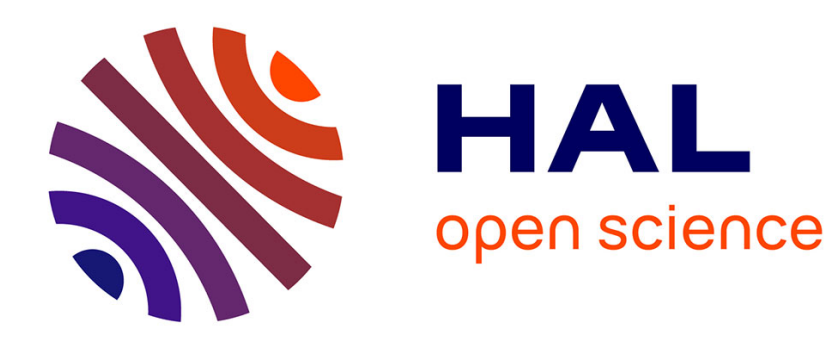

\title{
Electron microdiffraction reveals the nanoscale twist geometry of cellulose nanocrystals \\ Yu Ogawa
}

\section{To cite this version:}

Yu Ogawa. Electron microdiffraction reveals the nanoscale twist geometry of cellulose nanocrystals. Nanoscale, 2019, 10.1039/C9NR06044H . hal-02360161

\section{HAL Id: hal-02360161 \\ https://hal.science/hal-02360161}

Submitted on 14 Nov 2020

HAL is a multi-disciplinary open access archive for the deposit and dissemination of scientific research documents, whether they are published or not. The documents may come from teaching and research institutions in France or abroad, or from public or private research centers.
L'archive ouverte pluridisciplinaire HAL, est destinée au dépôt et à la diffusion de documents scientifiques de niveau recherche, publiés ou non, émanant des établissements d'enseignement et de recherche français ou étrangers, des laboratoires publics ou privés. 
1 Electron microdiffraction reveals the nanoscale twist geometry of cellulose nanocrystals

2

\section{Abstract}

8 systems.

$$
\text { Yu Ogawa }
$$

Univ. Grenoble Alps, CNRS, CERMAV, 38000 Grenoble, France

Nanocellulose consisting of crystalline cellulose nanoparticles has a high potential to serve as a building block for bio-based functional materials. The intrinsic chirality of cellulose crystals provides them with high added values such as optical properties and chiral induction ability. At the nanoscale, this chirality is connected to the right-handed longitudinal twisting of these fibrous crystallites. However, this nanoscale fibrillar twist has been a matter of debate due to contradictory data between ultrastructural observations and molecular simulations and so far, the exact twist geometry has not been elucidated. Here, an electron microdiffraction ( $\mu \mathrm{ED}$ ) analysis under cryogenic condition reveals the continuous twisting of cellulose nanocrystals (CNCs) in aqueous suspension. This intrinsic regular twist is drastically modified to discontinuous sharp twists when the CNCs are dried on flat surface. The present $\mu$ ED-based analysis at the single nanoparticle level allows establishing quantitative structure-properties relationship of various solid and colloidal nanocellulose

Keywords: Cellulose. Electron diffraction. Cryogenic transmission electron microscopy. Twist. Chirality. Nanoparticle. 
Introduction

Cellulose is attracting renewed scientific and industrial interests thanks to its high

27 potential as nanosized building blocks for functional bio-based materials. ${ }^{1-4}$ In this context, crystalline cellulose fibrils, commonly called nanocellulose, obtained from native cellulosic tissues, are categorized as short cellulose nanocrystals (CNCs) or long cellulose nanofibers (CNF). Together with their outstanding mechanical properties,,${ }^{5-9}$ one of the most fascinating aspects of nanocelluloses is their intrinsic chirality, which gives rise to various useful properties such as chiral optical activity ${ }^{10}$ and chiral induction ability. ${ }^{11}$ The chirality of nanocelluloses is present at various length scales, from the optically active glucosyl residues, i.e. the molecular building block of cellulose, to that of their suspensions, which are susceptible to spontaneously self-organize into chiral-nematic structures that can be preserved in a solid state after drying. ${ }^{10,12,13}$ At the nanometric scale, the chirality of cellulose can be observed as unidirectional twists along the fibrillar axes of nanocelluloses. ${ }^{14-}$ ${ }^{16}$ Such twists are of great importance since they are considered to govern the chiral-related properties of nanocellulose materials. ${ }^{1,11,17}$ Despite this, the ultrastructural details of the fibrillar twists of nanocelluloses have not been elucidated and have been in under debate due to contradicting experimental and computational observations as described below.

The twist of nanocelluloses has been observed using conventional imaging techniques such as atomic force microscopy (AFM), scanning and transmission electron microscopy (SEM and TEM, Fig. 1a-d) together with cryogenic TEM and electron tomography. ${ }^{14-16,18}$ Hanley and co-workers ${ }^{14}$ have reported ultrastructural details of the twisted morphology of large isolated nanofibrils extracted from the cell wall of the alga Micrasterias denticule dried on a flat substrate. In these, $180^{\circ}$ sharp right-handed twist regions occurred regularly spaced with flat and untwisted regions. ${ }^{14}$ Such sharp discontinuous right-handed twists, as illustrated

49 in Fig. 1e, were also reported for nanocelluloses extracted from other sources including wood 
and bacterial celluloses under similar observation conditions. ${ }^{15}$ These experimental

observations have been confronted with atomistic molecular dynamics (MD) simulations that also proposed right-handed twist occurrence for finite-size cellulose crystals. ${ }^{19-23}$ However, these MD simulations predict continuous smooth twists along the fiber axes (Fig. 1f) unlike the alternate occurrence of the sharp twists and flat untwisted regions observed experimentally. It remains to be determined whether the observed discontinuous twists in dried nanocellulose correspond to artifacts resulting from sample preparation or to intrinsic features of the samples.

To address the question of fundamental understanding of the twist morphology, one requires devising a methodology that allows quantitative description of local geometry of the cellulose crystal at the single nano-object level. In this work, a combination of cryogenic transmission electron microscopy (cryoEM) and electron microdiffraction ( $\mu \mathrm{ED})$ was used to follow the exact geometry of the twist of nanocelluloses as they occur in aqueous environment. Typically, a $\mu E D$ analysis can provide crystallographic information on individual nano-sized crystalline domains, thus leading to their local crystallographic orientation from two-dimensional diffraction patterns. By recording sequential $\mu$ ED patterns along the axis of cellulose nanocrystals embedded into vitreous ice, the structural details of the twist of CNCs, such as twist angle and pitch could be described. CNCs under dry and frozen conditions were compared, which allowed elucidating the effect of their drying on a supporting substrate.

\section{Results}

72 Sample

In this study, tunicate CNCs (tCNCs) extracted from the mantle of Halocynthia roretzi were used for their high crystallinity and large crystalline domains, hence their 
relatively high resistance toward electron radiation dose. The cross-section of a given $\mathrm{tCNC}$ consists in an elongated octagon where cellulose molecular sheets organized by hydrophobic interactions are stacked along its wider side (Supplementary Information, Fig. S1c) ${ }^{24}$ The basic structural characterization of the tCNCs used in this study is summarized in Fig. S2 in Supplementary Information file. Briefly, the tCNCs correspond to highly crystalline cellulose I $\beta$ crystals as described in literature. The average width such nanocrystals is estimated as 14.4 $\pm 3.3 \mathrm{~nm}$ based on the cryoTEM observations. The acid hydrolysis with sulfuric acid induced sulfate half-ester groups on the surface of the $\mathrm{tCNCs}$, giving a rise to negative surface charge of tCNCs. Such sulfate ester groups are detectable in FT-IR spectrum at $807 \mathrm{~cm}^{-1}$ (Fig. S2b). The zeta potential of $\mathrm{tCNC}$ was estimated as $-35.1 \mathrm{mV}$.

\section{Apparent fibrillar twist of cellulose nanocrystals visualized by TEM}

Figure 1a shows a cryoEM image of tCNCs in aqueous suspension where the twists are revealed as a gradual change in their contrast and their width along their length. They were also visible in tCNCs dried on a thin carbon film after negative staining (Fig. 1b) or metal shadowing (Fig.1d). The twists revealed in the images of shadowed tCNC are also righthanded and discontinuous as in the literature. The twist was virtually invisible in some short CNCs (Fig. 1c), being consistent with the low observation frequency of apparent twist, less than $10 \%$ in the report on twist morphology of wood and bacterial CNCs by Usov and coworkers. ${ }^{15}$

Although the twist is visible in these micrographs, the elucidation of the exact geometry of these twists is extremely challenging from the sole analysis of these images. This is partly because the cellulose crystals have an intrinsic size distribution in lateral dimensions, ${ }^{25-27}$ which precludes a direct correlation between their dimensions and their local internal structure. 

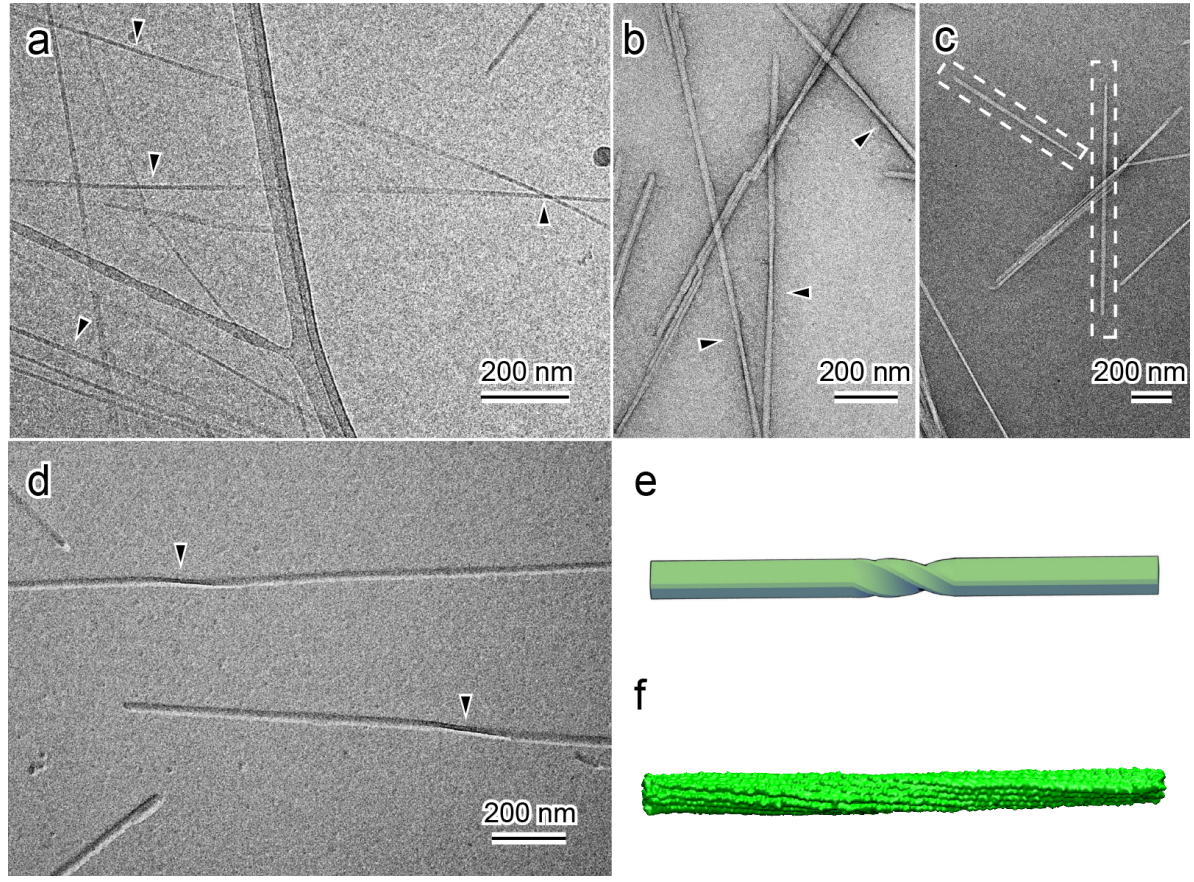

e

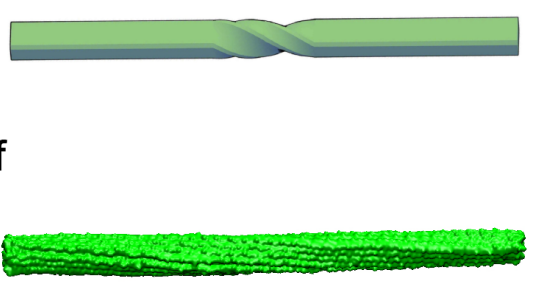

102 Figure 1. Twisted morphology of cellulose nanocrystals.

103 (a) CryoEM image of tCNCs; (b, c) conventional TEM image of negatively stained tCNCs;

104 (d) images of tCNCs shadowed with W/Ta alloy. In Fig. 1a-1d, the arrowheads point toward the apparent twisted regions, and dashed squares indicate apparently untwisted tCNCs. (e) schematized discontinuous twist morphology observed on CNCs in dry condition. (f) MDsimulated cellulose nanocrystal showing a right-handed twist.

\section{Intrinsic regular twist of tCNCs in aqueous suspension}

Fig. 2 summarizes the cryoEM/ $/ \mu \mathrm{ED}$ analysis of a single $\mathrm{tCNC}$ which was embedded in

111 vitreous ice, in such a way that its nanoscale morphology was maintained as in the aqueous

112 suspension state. These sets of two-dimensional $\mu$ ED patterns were recorded with an electron

113 probe of a diameter of approximately $70 \mathrm{~nm}$. The reflections in these patterns were indexed

114 according to the unit cell of cellulose I $\beta,{ }^{28,29}$ (Supplementary Information Figs. S1a and S1b), allowing the determination of the exact crystallographic orientation of the tCNC at each 
116 acquisition position with respect to the incident electron beam, as schematized in Figs S3 and

117 S4

118 As can be seen in Figure 2a, the first tCNC is relatively short with a length of

119 approximately $800 \mathrm{~nm}$ and isolated from other tCNCs and the supporting lacey carbon

120 network. Two diffraction patterns recorded at both ends show different projections, one

121 containing the $110^{*}$ vector perpendicular to the crystal direction and the other containing $b^{*}$

122 and $c^{*}$ indicating that this crystal is rotated along its longitudinal axis (Supporting

123 Information, Figure S3). Considering the right-handedness of the twist, comparison of the

124 two diffraction pattern is equivalent to the recording of another diffraction pattern with $45^{\circ}$

125 clock-wise rotation at point 1 . Thus, as previously demonstrated by diffraction experiment of

126 a series of rotation, ${ }^{30}$ the polarity of this $\mathrm{tCNC}$ was determined as having the c-axis pointing

127 upward in this image. Therefore, the reducing end of this crystal is located at its top-end since

128 the cellulose molecules are packed into a parallel-up manner in their unit cell. ${ }^{29,31}$ 
(a)

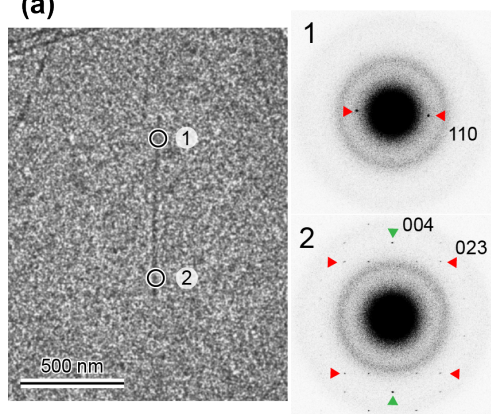

(b)

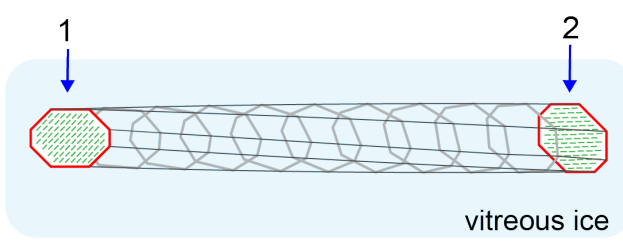

(c)
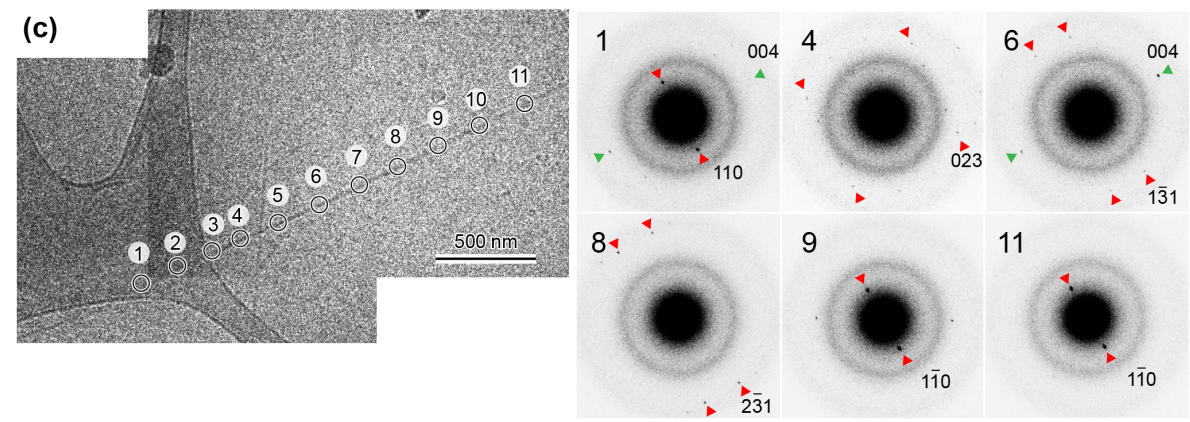

(d)

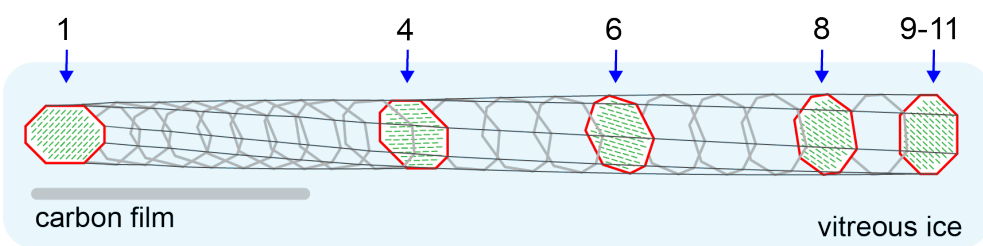

Figure 2. Longitudinal twist of tCNCs embedded in vitreous ice studied using $\mu$ ED.

132 (a, c) Diffraction contrast images of tCNCs under cryo-frozen condition, together with $\mu \mathrm{ED}$

133 patterns recorded from acquisition areas indicated by the circles. (b, d) Schematic

134 illustrations of the cross-sectional orientation of tCNC along their longitudinal axis at each acquisition point.

The second tCNC is longer and one of its ends is in contact with the carbon of the supporting lacey film (Figure 2c). For this tCNC, a series of $\mu \mathrm{ED}$ patterns were taken along the tCNC longitudinal axis over a $2.2-\mu \mathrm{m}$ distance at intervals of approximately $200 \mathrm{~nm}$.

140 Selected $\mu$ ED patterns are shown in Fig 2c 1-11 and the full diffraction data set is given in

141 Figure S3 together with schematic presentations of corresponding projections of reciprocal

142 lattice. The diffraction patterns at acquisition points 1-9 correspond to different projections of 143 the reciprocal lattice, indicating a continuous rotation of the crystal (Figs. 2d) with a rotation 
angle of $90^{\circ}$ from position 1 to 9 separated by $1.6 \mu \mathrm{m}$. On the other hand, the patterns from

145 positions 9-11 are identical, showing a projection containing the $1-10^{*}$ vector. As in the first example, the polarity of this $\mathrm{tCNC}$ can be determined considering the right handedness of

147 twist, indicating that the reducing end is located toward the left-hand end of the tCNC in the 148 figure.

149 As demonstrated by these $\mu$ ED analyses in Fig 2 together with other examples in Fig. S5, the tCNCs clearly possess the slow continuous twist along their longitudinal axes in the aqueous suspension state. Their twist rates along the longitudinal axis of the crystal are not constant not only between these two crystals but also within a single crystal itself as in the second tCNC. The twist rate of the first crystal between points 1 and 2 is of $5.8^{\circ} / 100 \mathrm{~nm}$

154 (Figure $2 \mathrm{~b}$ ), while the one of the second one is about $9 \% / 100 \mathrm{~nm}$ between positions 1 and 4 , and 4\%100 nm between positions 4 and 9, respectively (Figure 2d). The nanocrystals in Fig. S5 gave twist pitches of about 10\%100 nm (Fig. S5a) and 13\%/100 nm (Fig. S5b), respectively.

157 The difference between the twist geometries of these crystals is reasonable considering that 158 the tCNCs have a broad distribution in width and cross-sectional shape. ${ }^{25-27}$ MD simulations predict that the difference in cross-sectional shape of a cellulose crystal greatly affects its twist rate, where the crystal with a larger diameter should be less twisted. ${ }^{32}$ This effect is due to the contradictory contribution of the twisting tendency of individual chains and the elastic constraint against axial deformation that increases with distance to the center of the nanocrystal in the lateral plane. The change of twist rate in the second tCNC is presumably due to the fact that this tCNC is apparently in contact with the carbon film between points 1 and 3, which may cause a distortion of the twist geometry as a consequence of the interaction between cellulose and the carbon film. 
The $\mu$ ED experiments under cryogenic condition reveals the presence of intrinsic regular

170

171 twist of tCNCs in the aqueous suspension state as predicted in MD simulations (Fig. 1f). It is, however, incompatible with the apparent twist geometry observed in dry conditions (Fig. 1d and 1e). The similar $\mu \mathrm{ED}$ analysis was thus performed on a tCNC dried on a flat substrate, under a condition equivalent to those in the literature. ${ }^{14-16}$ Figure 3 a summarizes a sequential $\mu$ ED series obtained along a tCNC dried on an amorphous carbon film. The indexation of diffraction spots in Fig. 3a is given in Figure S6. The part of the tCNC subjected to this diffraction analysis shows a twist region separated by two flat segments. The flat segments give identical diffraction patterns, a projection of the reciprocal lattice containing $c^{*}$ and 11 $0^{*}$ vectors (Fig. 3a, positions $1-3$, and 5), so that the tCNC lays flat with its wider side flat on the carbon supporting film (Fig. 3b). The diffraction pattern obtained from the apparent twist region is a projection of planes containing $c^{*}$ and $a^{*}$ (Fig. 3a, position 4), indicating that the longer side of the octagonal cross-section was oriented diagonally with respect to the carbon film plane. This is consistent with the fact that the twisted region appeared to be thinner, compared to the flat segment regions. Thus, the tCNC is twisted by $180^{\circ}$ along its axis over the apparent twist position (Fig. 3b), the twist localized over a distance of less than $400 \mathrm{~nm}$ along the axis of the $\mathrm{tCNC}$, between positions 3 and 5 .

$$
\text { In this study, } 28 \text { other tCNCs dried on carbon films were analyzed using the same }
$$
sequential $\mu \mathrm{ED}$ measurements. Two examples of the analysis are given in Figs. S7 and S8 in the Supplementary Information file. All the nanocrystals possessed flat segments extending over more than $500 \mathrm{~nm}$. Among 42 observed flat segments, the majority (74\%) of them gave ED patterns of the projection of $110^{*}-c^{*}$ plane, while the other $26 \%$ were of the $120^{*}-c^{*}$ projection. 18 apparent sharp twist regions were examined. They provided the nanocrystals with either a $180^{\circ}\left(110^{*}-c^{*}\right.$ to $110^{*}-c^{*}$, Figs. 3 and S7) or $194^{\circ}$ flip $\left(110^{*}-c^{*}\right.$ to $120^{*}-c^{*}$, Fig. S8). No smaller twist angle was observed in this study. 
The difference of the twist geometries of the tCNCs under dry and cryo-frozen conditions is striking, as the tCNCs show a sharp localized twist when dried on a carbon film as opposed to a continuous extended twist in vitreous ice. The sharp twists observed under dry condition, not only in this study, but also in many images found in literature ${ }^{14-16}$ are thus a consequence of the drying process of CNCs on flat substrates, as suggested earlier by Hanley and coworkers. ${ }^{14}$ Considering that the surface tension between water and solid are significant at nanoscale, it is likely that the intrinsic twisting habit of cellulose nanocrystals is deformed by the large capillary force resulting from drying, with the result of the local de-twisting observed over the flat areas.

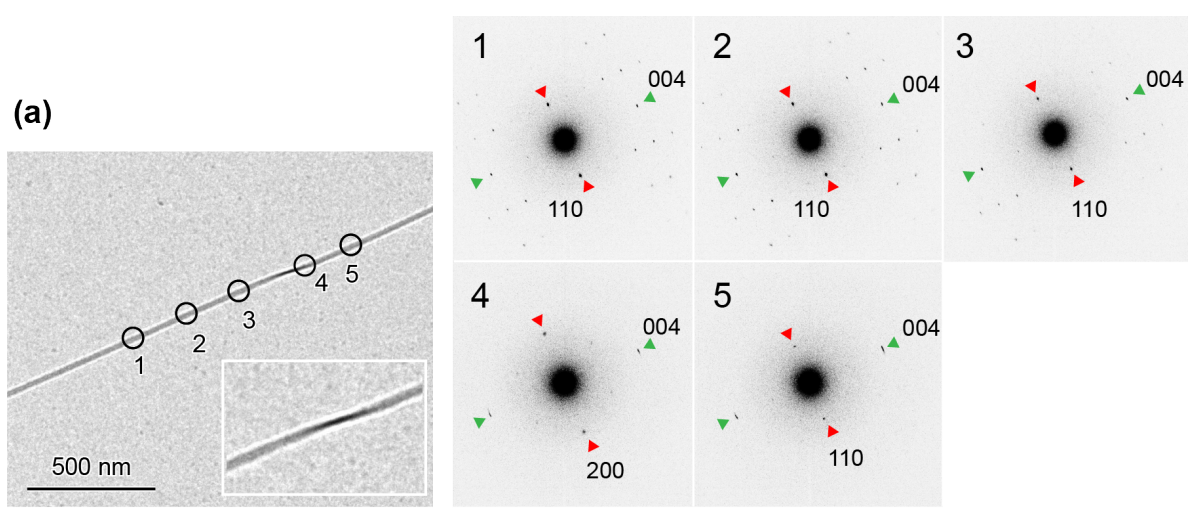

(b)

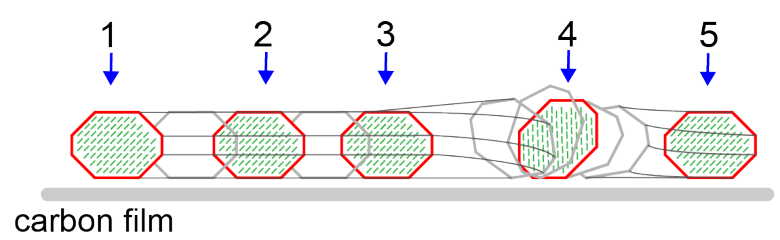

\section{Figure 3. Longitudinal twist of a dry tCNC followed by $\mu \mathrm{ED}$.}

(a) Diffraction contrast image of a tCNC dried on a carbon film, together with $\mu$ ED patterns recorded from the acquisition points indicated by circles. The diameter of the circle is equal to that of the electron probe used to record the patterns. Inset: enlarged image of the twisted region. (b) Schematic illustration of the cross-sectional orientation of the tCNC along longitudinal axis at each acquisition point. 
213 The use of $\mu \mathrm{ED}$ analysis is not only meaningful for single tCNCs, but is also extendable to

214 fibrillar bundles of nanocelluloses, commonly observed with various imaging techniques. ${ }^{15}$

215 Ultrastructural details of such assemblies are important as bundles of CNCs behave as single

216 particles in suspensions and thus have an impact on the suspension properties. In this line, it

217 is important to reveal (i) whether in a given bundle of parallel CNCs, the crystals have all the same polarity with respect to their reducing direction, (ii) whether the twisted regions may lead to the intertwinement of several CNCs, and (iii) whether the twists of individual crystallites are in sync.

Fig. 4a shows an assembly composed of two adjacent parallel tCNCs dried on a carbon film. As schematized in Fig. 4b, there is an apparent intertwinement of both tCNCs at their mid-lengths. $\mu$ ED patterns taken from a series of acquisition points along the tCNCs are shown in Fig 4c. The diffraction patterns obtained from the bottom half (Fig.4c, 1 and 2) correspond only to one projection containing $a^{*}$ and $c^{*}$ vectors, indicating that two crystals have the same cross-sectional orientation with respect to the incident electron beam. On the other hand, the patterns taken from positions 3 to 6 contain two different projections. The evolution of the cross-sectional orientation along the axis indicates that these two crystals have different rotation directions. Assuming that both crystals maintain their right handedness, these two crystals are therefore associated in an antiparallel manner with their crystal polarities being opposite to one another. In Figure 4a and its model in 4b, the "blue" nanocrystal has its reducing end toward the top of the pictures whereas in the "green" one, it is toward the bottom.

Based on this series of $\mu$ ED patterns, one can also deduce which of the surfaces of both crystals are in contact as illustrated in Fig. 4c. It is noticeable that the two crystals do not twist at the same location, but successively one after the other. Also, the contact planes 
between the two crystals are modified during the twisting, both crystals being jointed by their hydrophobic (200) planes in the locations 1 and 2, while at the end of the twist, in position 6 it is the (110) planes of one crystal, which is adjacent to the (200) plane of the other.

240

241 $300 \mathrm{~nm}$ : 2 : (b)

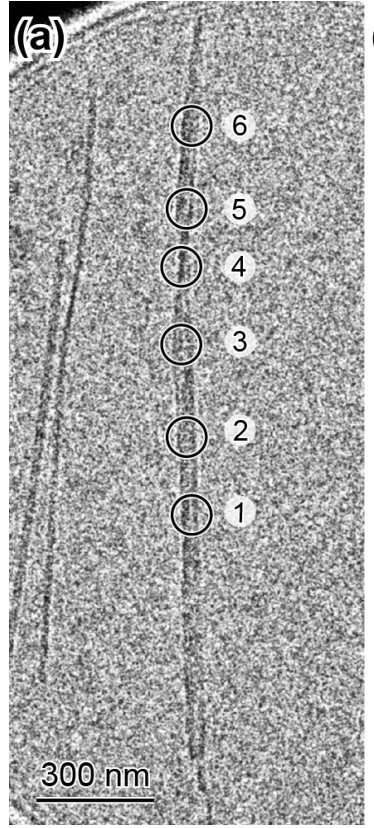

(c)

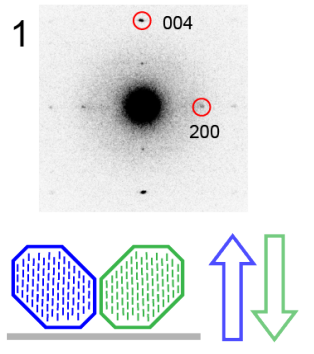

4
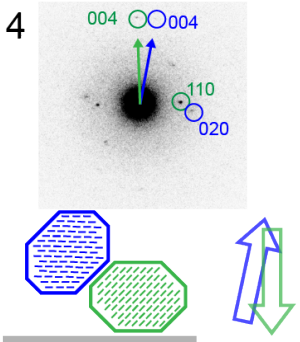

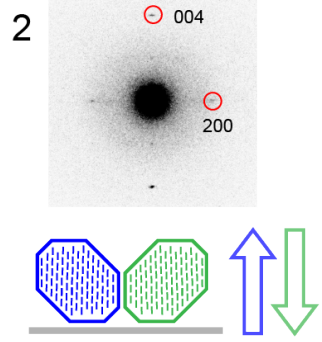

5

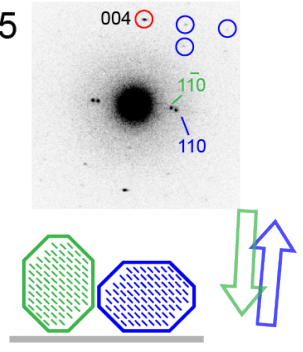

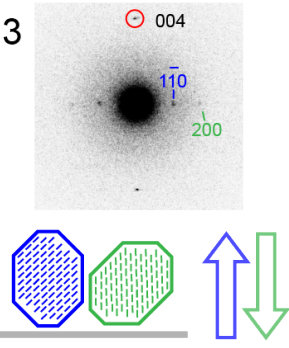

6

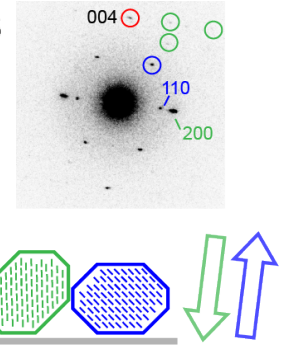

Figure 4. Joint assembly of two intertwined tCNCs. (a) Diffraction contrast image of the

joint assembly of two tCNCs. (b) Schematic illustration of the assembly. (c) Structural details

of the assembly at each acquisition point indicated in (a). Each subpanel contains a $\mu$ ED

pattern, a cross-sectional organization of both tCNCs, together with hollowed arrows

indicating the polarity of the tCNCs pointing toward their reducing ends. In $\mathrm{c} 4$, the solid arrows indicate that the directions of fiber $c$ axes are different for the two crystals.

\section{Discussion}

The comparison between the twist geometry of tCNCs under dry and cryo-frozen conditions highlights the importance of using cryoEM techniques to preserve the intact morphology of nanocelluloses in aqueous suspension. The effect of drying on the nanoscale morphology of tCNCs is significant, since it converts their initial regular longitudinal twist

254 into long flat untwisted segments alternating with sharp right-handed flips with a twist angle 
of about $180^{\circ}$. Although the twist morphology observed in the dried condition is ultimately derived from that in the suspension state, it remains to be investigated if and how these two morphologies are quantitatively correlated. Based on the amyloid fibril systems, it has been suggested that the adsorption of twisting nanofibers on a flat substrate would not alter the twist pitch when a full periodicity is observable even when the exact twist geometry was altered by the surface interactions with supporting substrates. ${ }^{33}$ However, this is often not the case for cellulose nanocrystal systems where the crystallite length can be shorter than its periodicity. In such a case the fibrillar twists are apparently suppressed due to the drying artifact as shown in Figure 1c. A systematic $\mu E D$ analysis with different surface properties of $\mathrm{CNC}$ and supporting substrates would be necessary to fully understand the influence of drying on the nanoscale morphology of such nanocrystals.

The drying on flat surface alters not only the twist geometry but also other nanoscale morphological features of nanocelluloses, such as the flexibility of these nanoelements. The occasional absence of the meridional 004 reflections was observed in these $\mu$ ED patterns recorded under cryo condition (Figure $2 \mathrm{~d}-4,8,9,11$ ) unlike those recorded under dry condition where the 004 reflection along the tCNC axis was always present (Figure 3a). The absence of this reflection indicates that the $c^{*}$ axis of the crystal is locally tilted with respect to a nearly flat Ewald sphere surface, hence, in the real space, the tCNC is not a straight rigid rod but rather consists in a somewhat wavy structure no longer flat in the vitrified ice plane.

274 This is surprising as especially short CNCs are often considered as rigid and straight bodies

275 thanks to their high axial elastic modulus. However, as recently reported, based on computational simulations, ${ }^{34,35}$ cellulose crystals can be considered as flexible in their

277 transverse and diagonal directions, which may lead to crystals deformation in such a waving manner along their axes. 

nanocrystals or nanofibrils consists in allowing the identification of the crystal polarity of

281 these elements without resorting to the reducing-end silver-labeling of the cellulose molecules, as described in previous studies. ${ }^{36}$ Determining the crystal polarity of cellulose crystals is important not only to characterize the assembled fibrillar structures composed of multiple cellulose crystallites, but also to understand the organization of cellulose in plant tissues. Indeed, during biosynthesis, the cellulose synthesizing complexes polymerize the nascent cellulose molecules from their non-reducing end and thus give its unique polarity to each nanofibril. ${ }^{30}$ Despite such unidirectional organization, adjacent cellulose nanofibrils have been characterized as statistically antiparallel in the cell wall of green algae, such as those of Valonia. ${ }^{36}$ This organization is likely due to the biogenesis of these nanofibrils, which are generated by slender synthesizing complexes of several hundred of $\mathrm{nm}$ in length, which themselves can align in a parallel or antiparallel manner in the plasma membrane of the corresponding alga. ${ }^{37}$ To date, one does not know whether such statistical distribution is common to the cellulose microfibrillar assembly in all plant cell walls, in particular to the more common ones where cellulose is being synthesized by the much smaller rosettes. The use of sequential $\mu \mathrm{ED}$ on bundle of nanofibrils pulled out from the corresponding plant cell wall could answer such questions.

The twisted structures described in this report correspond to tCNCs isolated from their environment. It is not clear whether these cellulose elements are also twisted in the native tunicate mantle from which they have been extracted. In this line, it is interesting to mention the case of $M$. denticulata cellulose as the observed twisting reported by Hanley et al. ${ }^{14}$ occurs only on isolated nanofibrils. In the cell wall of the corresponding alga, electron and Xray diffraction experiments have demonstrated that all nanofibrils are strictly laid flat in the algal cell wall with no hint of departure from the uniplanar orientation. ${ }^{38}$ This observation 
indicates that the inherent forces leading to the twist deformation of the nanofibrils can be

\section{$328 \quad$ Experimental}

\section{Conclusions}

easily counterbalanced by the surfaces forces resulting from the close contact with the neighboring nanofibrils as observed in the parallel assembly of tCNCs in this study (Fig. 4).

While it has been suggested that the twist morphology of cellulose nanofibrils in plant cell wall may hinder a coalescence of individual nanofibrils into merged fibrils, ${ }^{22,39}$ the microdiffraction study shown here for the model samples suggests that the twisting do not avoid tight adhesion of adjacent microfibrils on non-matching crystallographic surfaces. The persistence of the twisted structures in-planta remains to be investigated.

This report demonstrates that the $\mu \mathrm{ED}$ analysis allows quantitatively describing the nanoscale structural features of nanocelluloses. The exact geometry of intrinsic continuous twist of tCNC in aqueous suspension state was revealed for the first time under cryogenic condition. With dried samples, this regular twist was significantly altered into discontinuous sharp twist separated by long flat segments, giving rise to the discrepancy between the apparent morphology obtained from conventional TEM and AFM imaging and molecular simulations of CNCs. The similar $\mu \mathrm{ED}$ analysis applied to a fibillar assembly of tCNCs provided the structural details of this common structural unit of nanocellulose systems, namely the molecular polarities and twist rates of constituent nanocrystals as well as interacting surfaces between crystals. The quantitative structural description at single nanoparticle level leads to establish the structure-properties relationship of various solid and colloidal nanocellulose systems. Such knowledge will pave a way to use the renewable cellulose for rationally designing specific biobased functional materials. 
329 Sample preparation. Cellulose nanocrystals (CNCs) were prepared according to the method

330

331

332

333

334

335

336

337

338

339

340

341

342

343

344

345

346

347

348

349

350

351

352

353 described by Elazzouzi-Hafraoui et al. ${ }^{26}$ Briefly, Halocynthia roretzi tunicin was purified by alternating aqueous treatments with $1 \mathrm{~N} \mathrm{KOH}$ and $0.3 \% \mathrm{NaClO}_{2}$ at room temperature followed by hydrolysis with $48 \%$ sulfuric acid at $55{ }^{\circ} \mathrm{C}$ for $15 \mathrm{~h}$. Acid-free $\mathrm{CNCs}$ aqueous suspensions were obtained after repeated centrifugations/washing cycles followed by ultrasonication.

Structural characterization of tCNC. X-ray diffraction measurement was carried out on a drid film of tCNC with Ni-filtered CuK $\alpha$ radiation $(\lambda=0.15418 \AA)$ using a Philips PW3830 generator operated at $30 \mathrm{kV}$ and $20 \mathrm{~mA}$. FTIR measurement was performed with the same dried tCNC film using a Spectrum Two spectrometer (Perkin Elmer) operated under attenuated total reflection (ATR) mode. The zeta potential was measured by electrophoresis coupled with laser Doppler velocimetry using a Malvern NanoZS instrument. The $0.1 \mathrm{wt} \%$ tCNC suspension was measured at $\mathrm{pH} 7$ and with $0.01 \mathrm{M} \mathrm{NaCl}$. Data were averaged over three measurements.

Transmission electron microscopy (TEM). Drops of about $0.001 \mathrm{wt} \% \mathrm{CNC}$ aqueous suspensions were deposited on glow-discharged carbon-coated copper grids. Negative staining was achieved using $2 \mathrm{wt} \%$ uranyl acetate aqueous solution. Metal shadowing was performed with a BAL-TEC MED 020 coating system (Leica microsystems, Germany), using tungsten/tantalum alloy sputtered from an electron gun, with a shadowing angle of $30^{\circ}$. Specimens for observations under cryogenic conditions were prepared by quench-freezing in liquid ethane by using an automatic plunge freezer (EM-GP, Leica Microsystems, Germany). Transmission electron microscopy was performed using a JEM-2100Plus (JEOL Ltd., Japan) operated at an accelerating voltage at $200 \mathrm{kV}$. All electron micrographs and electron 
diffraction patterns were recorded on a Gatan Rio 16 camera (Gatan Inc., USA). An Elsa cryo-transfer holder (Gatan Inc., USA) was used for cryoEM observations. Low-dose brightfield imaging and microED $(\mu E D)$ measurements were achieved using the SerialEM program. ${ }^{40}$ Micrographs and $\mu$ ED patterns were analyzed using the Fiji program. ${ }^{41}$

Molecular dynamics (MD) simulation. MD simulation of cellulose nanocrystals was performed using Gromacs version $5^{42}$ and Gromos 56Acarbo force field. ${ }^{43}$ The nanocrystals were composed of 18 molecular chains having 80 glucose residues. The details of the simulation are described elsewhere. ${ }^{34}$

363

\section{Conflicts of interest}

365

There are no conflicts to declare.

Acknowledgements

I would like to thank Drs. H. Chanzy, Y. Nishiyama, K. Mazeau, J-L Putaux, and S.

Vignolini for suggestions during the writing of this work. I also would like to thank Drs.

Bruno Jean, Christine Lancelon-Pin and Pierre Saillar for their help in zeta-potential measurement, metal shadowing, and drawing of schematic illustration. The NanoBio-ICMG platform (FR 2607) is acknowledged for granting access to the electron microscopy facility.

\section{Supplementary Information}

374 Supplementary information file is available online.

\section{References}

377 1. Y. Habibi, L. A. Lucia, and O. J. Rojas, Chem. Rev. 110, 3479-3500 (2010). 
378 2. D. Klemm, B. Heublein, H-P. Fink, and A. Bohn, Angew. Chem. Int. Edit. 44, 3358$3793393(2005)$.

380 3. R. J. Moon, A. Martini, J. Nairn, J. Simonsen, and J. Youngblood, Chem. Soc. Rev. $38140,3941-3994(2011)$.

382 4. A. Isogai, J. Wood Sci. 59, 449-459 (2013).

383 5. I. Sakurada, Y. Nukushina, and T. Ito, J. Polym. Sci. 37, 651-660 (1962).

384 6. T. Nishino, K. Takano, and K. Nakamae, J. Polym. Sci. Part B Polym. Phys. 33, $385 \quad 1647-1651(1995)$.

386 7. I. Diddens, B. Murphy, M. Krisch, and M. Müller, Macromolecules 41, 9755-9759 387 (2008).

$388 \quad 8 . \quad$ Y. Nishiyama, J. Wood Sci. 55, 241-249 (2009).

389 9. T. Saito, R. Kuramae, J. Wohlert, L. A. Berglund, and A. Isogai, Biomacromolecules 390 14, 248-253 (2013).

391 10. B. D. Wilts, A. G. Dumanli, R. Middleton, P. Vukusic, and S. Vignolini, $A P L$ 392 Photonics 2, 040801 (2017).

393 11. M. Kaushik, K. Basu, C. Benoit, C. M. Cirtiu, H. Vali, and A. Moores, J. Am. Chem. 394 Soc. 137, 6124-6127 (2015).

395 12. J-F. Revol, H. BradfordJ. Giasson, R. H. Marchessault, and D. G. Gray, Int. J. Biol. 396 Macromol. 14, 170-172(1990).

397 13. R. M. Parker, G. Guidetti, C. A. Williams, T. Zhao, A. Narkevicius, S. Vignolini, and 398 B. Frka-Petesic, Adv. Mater. 30, 1704477 (2018).

399 14. S. J. Hanley, J-F. Revol, L. Godbout, and D. G. Gray, Cellulose 4, 209-220 (1997).

400 15. I. Usov, G. Nyström, J. Adamcik, S. Handschin, C. Schütz, A. Fall, L. Bergström, and 401 R. Mezzenga, Nat. Commun. 6, 7564-1-7564-11 (2014). 
402

403

404

405

406

407

408

409

410

411

412

413

414

415

416

417

418

419

420

421

422

423

424 (1990).

425 (1998).

16. M. Arcari, E. Zuccarella, R. Axelrod, J. Adamcik, A. Sánchez-Ferrer, R. Mezzenga, and G. Nyström, Biomacromolecules (2019).

17. W. J. Orts, L. Godbout, R. H. Marchessault, and J-F. Revol, Macromolecules 31, $5717-5725(1998)$.

18. J. Majoinen, J. Haataja. D. Appelhans. A. Lederer, A. Olszewska, J. Seitsonen, V. Aseyev, E. Kontturi, H. Rosilo, M. Österberg. N. Houbenov, and O. Ikkala. J. Am. Chem. Soc. 136, 865-869 (2013).

19. J. F. Matthews, C. E. Skopec, P. E. Mason, P. Zuccato, R. W. Torget, J. Sugiyama, M. W. Himmel, and J. W. Brady, Carbohydr. Res. 341, 138-152 (2006).

20. S. Paavilainen, T. Rog, and I. Vattulainen, J. Phys. Chem. B 115, 3747-3755 (2011).

21. J. A. Hadden, A. D. French, and R. J. Woods, Biopolymers 99,746-756 (2013).

22. L. Bu, M. E. Himmel, and M. F. Crowley, Carbohydr. Polym. 125, 146-152 (2015).

23. S. K. Kannan, D. P. Oehme, M. S. Doblin, M. S. Gidley, A. Bacik, and M. T. Dowton, Carbohydr. Polym. 175, 433-439 (2017).

24. W. Helbert, Y. Nishiyama, T. Okano, and J. Sugiyama, J. Struct. Biol. 124, 42-50

25. Y. Van Daele, J-F. Revol, F. Gaill, and G. Goffinet, Biol. Cell. 76, 87-96 (1992).

26. S. Elazzouzi-Hafraoui, Y. Nishiyama, J-L. Putaux, L. Heux, F. Dubreuil, and C. Rochas, Biomacromolecules 9, 57-65 (2008).

27. B. Frka-Petesic, J. Sugiyama, S. Kimura, H. Chanzy, and G. Maret, Macromolecules 48, 8844-8857 (2015).

28. J. Sugiyama, T. Okano, H. Yamamoto, and F. Horii, Macromolecules 23, 3196-3198

29. Y. Nishiyama, P. Langan, and H. Chanzy, J. Am. Chem. Soc. 124, 9074-9082 (2002). 
426 30. M. Koyama, W. Helbert, T. Imai, J. Sugiyama, and B. Henrissat, Proc. Natl. Acad. 427 Sci. U.S.A. 94, 9091-9095 (1997).

428 31. A. Sarko, and R. Muggli, Macromolecules 7, 486-494 (1974).

429 32. Z. Zhao, O. E. Shklyaev, A. Nili, M. N. A. Mohamed, J. D. Kubicki, V. H. Crespi, 430 and L. Zhong, J. Phys. Chem. A 117, 2580-2589 (2012).

431 33. S. Assenza, J. Adamcik, R. Mezzenga, and P. De Los Rios, Phys. Rev. Lett. 113, $432 \quad 268103(2014)$.

433 34. P. Chen, Y. Ogawa, Y. Nishiyama, A. E. Ismail, and K. Mazeau, Phys. Chem. Chem. 434 Phys. 18, 19880-19887 (2016).

435 35. G. Molnar, D. Rodney, F. Martoïa, P. J. J. Dumont, Y. Nishiyama, K. Mazeau, and L. 436 Orgeas, Proc. Natl. Acad. Sci. U.S.A. 115, 7260-7265 (2018).

437 36. N-H. Kim, T. Imai, M. Wada, and J. Sugiyama, Biomacromolecules 7, 274-280 438 (2006).

439 37. T. Itoh, and R. M. Brown, Planta 160, 372-381 (1984).

440 38. N-H. Kim, W. Herth, R. Vuong, and H. Chanzy, J. Struct. Biol. 117, 195-203 (1996).

441 39. A. N. Fernandes, L. H. Thomas, C. M. Altaner, P. Callow, V. T. Forsyth, D. C.

442 Apperley, C. J. Kennedy, and M. C. Jarvis, Proc. Natl. Acad. Sci. U.S.A. 108, 1195-1203 $443(2011)$.

444 40. D. N. Mastronarde, Microsc. Microanal., 9, 1182-1183 (2003).

445 41. J. Schindelin, I. Arganda-Carreras, I. E. Frise, et al. Nat. Methods 9, 676-682 (2012).

446 42. M. J. Abraham, T. Murtola, R. Schulz, S. Páll, J. C. Smith, B. Hess, and E. Lindahl, 447 SoftwareX, 1, 19-25(2015).

448 43. H. S. Hansen, and P. H. Hünenberger, J. Comp. Chem. 32, 998-1032 (2011). 